\title{
Impact of Reflections in Enclosed mmWave Wearable Networks
}

\author{
Geordie George and Angel Lozano \\ Universitat Pompeu Fabra (UPF) \\ 08018 Barcelona, Spain \\ Email:\{geordie.george, angel.lozano\}@upf.edu
}

\begin{abstract}
We study the impact of signal reflections in enclosed wireless networks of wearable devices operating at mmWave frequencies. Given the radical blockage by obstacles and people (including the user's own body) at these frequencies, surface reflections are expected to be very important contributors to the collection of an adequate amount of desired signal power. At the same time, they are also expected to substantially increase the level of interference reaching any given receiver. Our objective is to understand the interplay of these two effects in relevant enclosed settings with high user densities (e.g., commuter trains, subways, airplanes, airports, or offices) in order to help assess the viability of mmWave operation in such settings.
\end{abstract}

\section{INTRODUCTION}

With the advent of wearable wireless technology [1], people may soon be featuring multiple mobile communication devices on and around their bodies. There is therefore interest in establishing the feasibility of deploying very dense wearable networks, chiefly in enclosed settings [2], [3] via device-todevice (very short range) links and preferably at mmWave frequencies [4]. Moreover, as the form factor and cost of these small devices may constrain the antenna designs, it is interesting to evaluate the practicality of simple antennas.

At mmWave frequencies, signals exhibit minimal scattering or diffraction around blocking obstacles, but strong specular reflections off surfaces [2], [5], [6] and hence these reflections are expected to play a major role in the performance of mmWave enclosed networks. The bodies of the users themselves can act as blockages [3], [6], [7].

In this paper, we investigate the impact of reflections and user blockages on the fundamental performance limits of an enclosed mmWave network with emphasis on dense deployments of single-antenna wearables. Building on [3], where human body blockages in direct links were modeled explicitly but reflections were not, we incorporate the reflections off interior surfaces and account for blockages in both direct and reflected paths. A stochastic blockage model is derived for users uniformly distributed within the enclosed space, which helps obtain results without the need to exhaustively test whether each individual link is blocked. Ultimately, we seek to understand whether reflections are beneficial or detrimental, and whether satisfactory performance is possible in relevant enclosed settings.

This work was supported in part by Intel's University Research Program "5G: Transforming the Wireless User Experience" and by the MINECO Project TEC2012-34642.

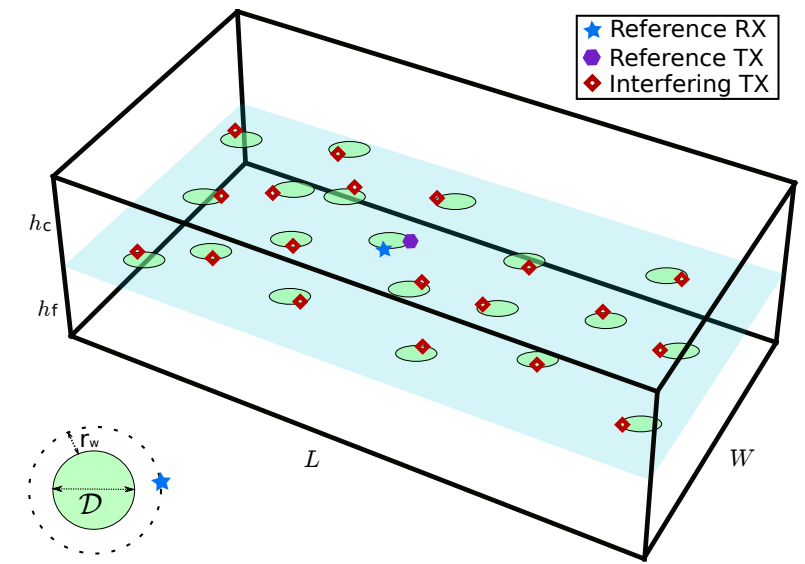

Fig. 1. Co-channel wearable devices on the users in an enclosed space.

\section{NeTwORK MODEL}

Consider users within an enclosed space. Each user wears multiple communication devices and the intended transmissions are always between devices on a same user. The transmissions on each user are assumed orthogonal as they can be co-ordinated via a hub on the user. Thus, interfering transmissions are always from wearables on other users.

We focus on a time-frequency channel occupied by a reference transmitter-receiver pair on a reference user. Besides the reference user, there are $K$ other users on which the interfering transmitters reusing the same channel are located. Each user has one transmitter on the channel under consideration.

\section{A. Network Geometry}

The reference receiver and all the co-channel transmitters lie on a horizontal plane, denoted by $\mathcal{X}$, at a height $h_{\mathrm{c}}$ below the ceiling and $h_{\mathrm{f}}$ above the floor, within an enclosed space shaped as an $L \times W \times\left(h_{\mathrm{c}}+h_{\mathrm{f}}\right)$ cuboid (cf. Fig. 1). The users are modeled as circles of diameter $\mathcal{D}$ on $\mathcal{X}$. Each wearable is oriented randomly in $[0,2 \pi)$ and at distance $\frac{\mathcal{D}}{2}+r_{\mathrm{w}}$ from its circle center, where $r_{w}$ represents the distance of each wearable from its user's body. The reference receiver is located at $\mathrm{X}_{0}$ while the $K+1$ transmitters are located at $\left\{X_{k}\right\}_{k=0}^{K}$, with $X_{0}$ the reference transmitter. Note how we utilize different fonts to distinguish between the receiver and the transmitters. The distance between $X_{k}$ and $\mathrm{X}_{0}$ is $r_{k}=\left\|X_{k}-\mathrm{X}_{0}\right\|$. 


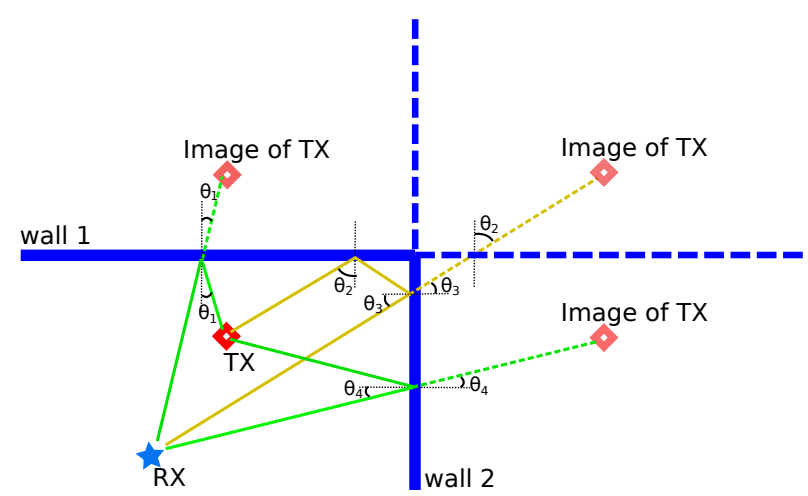

Fig. 2. Reflected links from a transmitter to a receiver, off two walls. There are two first order reflections and one second order reflection.

\section{B. Reflections}

The transmission from $X_{k}$ reaches $\mathrm{X}_{0}$ via the direct propagation link between them and via the reflections off the interior surfaces. In order to model these reflections, we need the lengths of the reflected links as well as the angles of incidence. Adding extra (phantom) transmitters at the mirror image locations across each surface (cf. Fig. 2) facilitates the reflection modeling.

In this paper, we consider only first-order reflections (single bounces off each surface). From each transmitter $X_{k}$ there are six such reflected links reaching $X_{0}$, which are incorporated by adding six image transmitters. The four walls are indexed with $i=1, \ldots, 4$, the ceiling with $i=5$, and the floor with $i=6$. For $i=1, \ldots, 6$, the images of $X_{k}$ are located at $X_{i, k}$, the corresponding angles of incidence are $\theta_{i, k}$, and the reflected link distances are $r_{i, k}=\left\|X_{i, k}-\mathrm{X}_{0}\right\|$. The coordinates of the image locations and the angles of incidence can be easily obtained as functions of $X_{k}$ and $\mathrm{X}_{0}$, as detailed in [8, Appendix A].

The reflectivity of a surface depends on some physical properties of the material and on the angle of incidence. In this paper, all six surfaces have the same physical properties.

Note that the links from $\left\{X_{i, 0}\right\}_{i=1}^{6}$ correspond to the reflections of the intended transmission from $X_{0}$. While the intended transmission has a direct on-body link and six reflected offbody links, all the interfering links (both direct and reflected) are off-body.

\section{Body Blockages}

The links between wearables (both direct and reflected) can get blocked by the users' bodies. Since the reflections are modeled explicitly, and the penetration losses at mmWave frequencies are very high, we assume that no signal traverses such blockages. The blocking of the direct link from $X_{k}$ is indicated by a binary variable $\beta_{k}$, which equals 1 for unblocked and 0 for blocked. Likewise, the blocking of the link from $X_{k}$ reflected off the $i$ th surface is indicated by another binary variable $\beta_{i, k}$.

As in [3], the direct path between $X_{k}$ and $\mathrm{X}_{0}$ is considered blocked if it intersects any of the circles; however, unlike in
[3], we do not ignore self-body blockages [9], i.e., the link between $X_{k}$ and $\mathrm{X}_{0}$ can get blocked by the transmitting or receiving user body. Applied to the corresponding images, this blockage model further extends to the reflected links off the four walls and an algorithm for determining such blockages is given in [8, Appendix B].

Given the significant separation between the ceiling and the users' bodies, we assume that the reflections off the ceiling are never blocked, i.e., $\beta_{5, k}=1$. And, as the users' bodies touch the floor, each reflection off the floor gets blocked only if the corresponding direct path is blocked, i.e., $\beta_{6, k}=\beta_{k}$.

The foregoing blockage model is applicable to all the offbody links. As for the on-body intended link, since it is between the wearables on the same user it should have an independent blockage/shadowing model. In the absence of a good model for on-body shadowing, we consider two specific cases: unblocked on-body link $\left(\beta_{0}=1\right)$ and blocked on-body link $\left(\beta_{0}=0\right)$. When the on-body link is blocked, transmission from $X_{0}$ reaches $\mathrm{X}_{0}$ only via the reflected links. The blocking of the reflections off the four walls follow the blockage model, while the ones off the ceiling and the floor are assumed to be unblocked $\left(\beta_{5,0}=\beta_{6,0}=1\right)$. A possible future refinement for $\beta_{5,0}$ and $\beta_{6,0}$ would be to model them based on specific wearable applications.

\section{PRopagation Model}

All wearables feature a single antenna and the (fixed) transmit power is denoted by $P$. The transmission from $X_{k}$ is received at $\mathrm{X}_{0}$ with power

$$
P_{k}=\mathrm{L}\left|\beta_{k} r_{k}^{-\frac{\eta}{2}}+\sum_{i=1}^{6} \beta_{i, k} r_{i, k}^{-\frac{\eta}{2}} \Gamma\left(\theta_{i, k}\right) e^{-\mathrm{j} \Delta \phi_{i, k}}\right|^{2} P
$$

where $\mathrm{L}$ is the pathloss at $1 \mathrm{~m}, \eta$ is the pathloss exponent, $\Gamma(\cdot)$ is the reflection coefficient and $\Delta \phi_{i, k}=2 \pi\left(r_{i, k}-r_{k}\right) / \lambda$ is the phase difference between the direct link and the $i$ th reflected link. As the operating wavelength $\lambda$ is in the order of millimeters, $\Delta \phi_{i, k}$ varies widely even with small changes in the path difference $\left(r_{i, k}-r_{k}\right)$. Due to the simplifying assumption that all wearables are on a horizontal plane, the model fails to capture the phase changes caused by the likely altitude variations of the wearable locations. Therefore, so as to compensate for the invariability imposed by this assumption, the phase differences corresponding to the ceiling and floor reflections $\left(\Delta \phi_{5, k}\right.$ and $\left.\Delta \phi_{6, k}\right)$ are drawn uniformly at random in $[0,2 \pi)$. Recall that $\left\{\beta_{k}\right\}$ and $\left\{\beta_{i, k}\right\}$ are determined as explained in the previous section, slightly differently for the intended links $(k=0)$ and the interfering links $(k=1, \ldots, K)$.

\section{LOCAL-AVERAGE SINR}

The local-average SINR (signal-to-interference-plus-noise ratio) at the reference receiver is given by

$$
\rho=\frac{P_{0}}{\sigma^{2}+\sum_{k=1}^{K} P_{k}}
$$

where $\sigma^{2}=F_{\mathrm{N}} N_{0} B$ is the AWGN power, with $F_{\mathrm{N}}$ the receiver noise figure, $N_{0}$ the noise power spectral density, and $B$ the bandwidth. 


\section{Stochastic Blockage Model}

From the user distribution on $\mathcal{X}$, the blockage probabilities of off-body links can be computed in order to devise, as an alternative to the algorithm presented in [8, Appendix B] to determine blockages, a stochastic model for those blockages. The blockage probabilities are computed using results from random shape theory [10]. For a given $\mathrm{X}_{0}$, we consider that $\left\{X_{k}\right\}_{k=1}^{K}$ conform to a uniform binomial point process (BPP) on $\mathcal{X}$, i.e., $K$ independent and identically distributed (i.i.d.) points on $\mathcal{X}$ with density $\frac{1}{L W}$. Then, the probability of a given off-body link being blocked can be obtained as function of its link length.

For the direct interference link from a given transmitter $X_{k}$ to $\mathrm{X}_{0}$, considering the potential blockages by the $K-1$ other users and the potential self-body blocking by the link's own users (the reference user and the user of $X_{k}$ ), the probability of blockage is obtained as (cf. [8, Appendix C])

$$
\begin{aligned}
p_{\mathrm{B}_{\mathrm{I}}}\left(r_{k}\right)=1-(1- & \left.\frac{r_{k} \mathcal{D}+\pi \mathcal{D}^{2} / 4}{L W}\right)^{K-1} \\
& \cdot\left(1-\frac{\arcsin \frac{\mathcal{D}}{2 \mathrm{r}_{\mathrm{w}}+\mathcal{D}}}{\pi}\right)^{2}
\end{aligned}
$$

and we model $\beta_{k}$, for each given $r_{k}$, as a Bernoulli random variable with $\mathbb{P}\left[\beta_{k}=0\right]=p_{\mathrm{B}_{\mathrm{I}}}\left(r_{k}\right)$.

As shown in [8, Appendix C], the probability of blockage of the reflected interfering links off the walls, i.e., the links from $\left\{X_{i, k}\right\}_{i=1}^{4}$, can also be approximated by (3). Thus, for $i=$ $1, \ldots, 4, \beta_{i, k}$ is (for each given $r_{i, k}$ ) Bernoulli with $\mathbb{P}\left[\beta_{i, k}=\right.$ $0] \approx p_{\mathrm{B}_{\mathrm{I}}}\left(r_{i, k}\right)$.

As for the signal reflections off the walls, i.e., the links from $\left\{X_{i, 0}\right\}_{i=1}^{4}$, only the reference user can effect self-body blockage on them while the other $K$ users can potentially intersect the links. Then, as argued in [8, Appendix C], the probability of blockage for the link from $X_{i, 0}$ can be approximated as

$$
\begin{aligned}
& p_{\mathrm{B}_{\mathrm{S}}}\left(r_{i, 0}\right) \approx 1-(1-\left.\frac{r_{i, 0} \mathcal{D}+\pi \mathcal{D}^{2} / 4}{2 L W}\right)^{K} \\
& \cdot\left(1-\frac{\arcsin \frac{\mathcal{D}}{2 r_{\mathrm{w}}+\mathcal{D}}}{\pi}\right)
\end{aligned}
$$

and, for $i=1, \ldots, 4, \beta_{i, 0}$ is hence modeled (given $r_{i, 0}$ ) as Bernoulli with $\mathbb{P}\left[\beta_{i, 0}=0\right]=p_{\mathrm{B}_{\mathrm{S}}}\left(r_{i, 0}\right)$.

From the marginal distributions for $\left\{\beta_{k}\right\}$ and $\left\{\beta_{i, k}\right\}$ established above, a stochastic blockage model can be constructed by regarding these variables as independent, functions only of their respective link lengths. This stochastic model ignores the inevitable dependences across links due to common blockages and related reflections, and thus it requires validation.

\section{EXAMPLES}

Next, we provide examples under the system settings in Table I (where $\eta$ and $\mathrm{L}$ are chosen in accordance with freespace pathloss at $60 \mathrm{GHz}$ ) to test the accuracy of the stochastic blockage model proposed in Section $\mathrm{V}$ and to gauge the impact
TABLE I

SySTEM SETTINGS

\begin{tabular}{|c|c|c|c|}
\hline Parameter & Value \\
\hline$L$ & $20 \mathrm{~m}$ \\
\hline$W$ & $4 \mathrm{~m}$ \\
\hline$h_{\mathrm{c}}$ & $1 \mathrm{~m}$ \\
\hline$h_{\mathrm{f}}$ & $1 \mathrm{~m}$ \\
\hline $\mathcal{D}$ & $50 \mathrm{~cm}$ \\
\hline $\mathrm{r}_{\mathrm{w}}$ & $10 \mathrm{~cm}$ \\
\hline$r_{0}$ & $25 \mathrm{~cm}$ \\
\hline
\end{tabular}

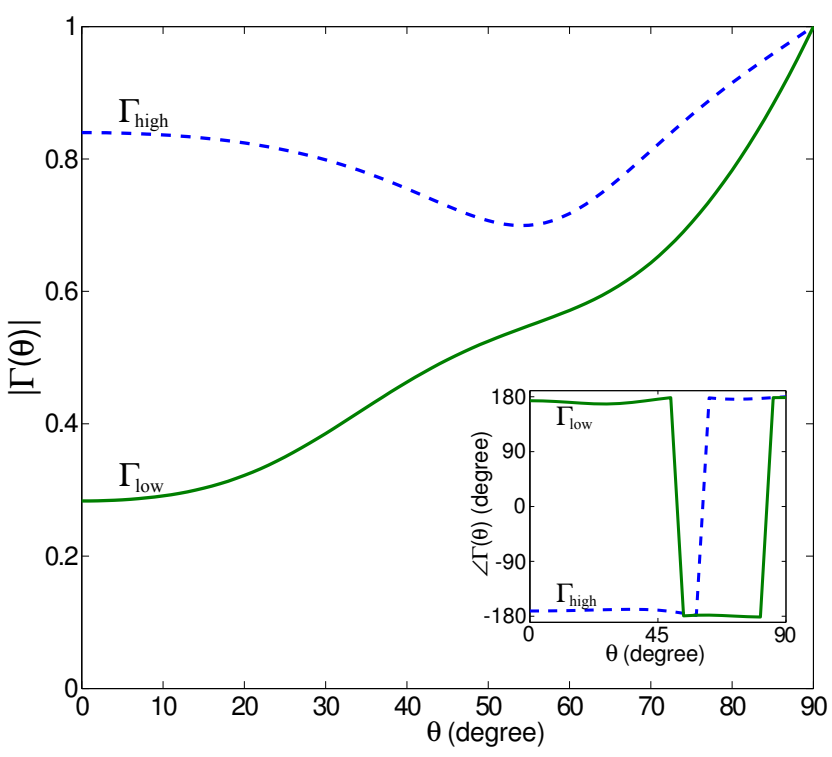

Fig. 3. Magnitude (main plot) and phase (inset plot) of low $\left(\Gamma_{\text {low }}\right)$ and high $\left(\Gamma_{\text {high }}\right)$ reflection coefficients as functions of the angle of incidence $\theta$.

of reflections. Results are obtained for two specific locations for the reference receiver $\mathrm{X}_{0}$ : Center of $\mathcal{X}$ and Corner of $\mathcal{X}$ ( $9 \mathrm{~m}$ along the length and $1.5 \mathrm{~m}$ along the width).

As for the reflectivity of the surfaces, we consider the two coefficients illustrated in Fig. 3: low reflectivity $\Gamma_{\text {low }}$ and high reflectivity $\Gamma_{\text {high }}[8$, Appendix D]. Both correspond to a homogeneous dielectric plate with a smooth surface at 60 $\mathrm{GHz}$, but with different values for thickness and refractive index [11].

Example 1. For a reference receiver located at the center and $K=30$ interferers, the CDFs of the local-average SINR $\rho$ are plotted in Fig. 4. The CDFs obtained by independently realizing $\left\{\beta_{k}\right\}$ and $\left\{\beta_{i, k}\right\}$ via the probabilities given in Section $\mathrm{V}$, ignoring their dependences, are contrasted against the exact ones obtained by establishing each individual blockage deterministically as detailed in [8, Appendix B].

Very good agreements are observed between the various pairs of CDFs in Fig. 4, supporting the stochastic model under these settings.

Given the minimal fading at mmWave frequencies, the spectral efficiency (bits/s/Hz) can be obtained from $\rho$ as

$$
C(\rho)=\log _{2}(1+\rho)
$$




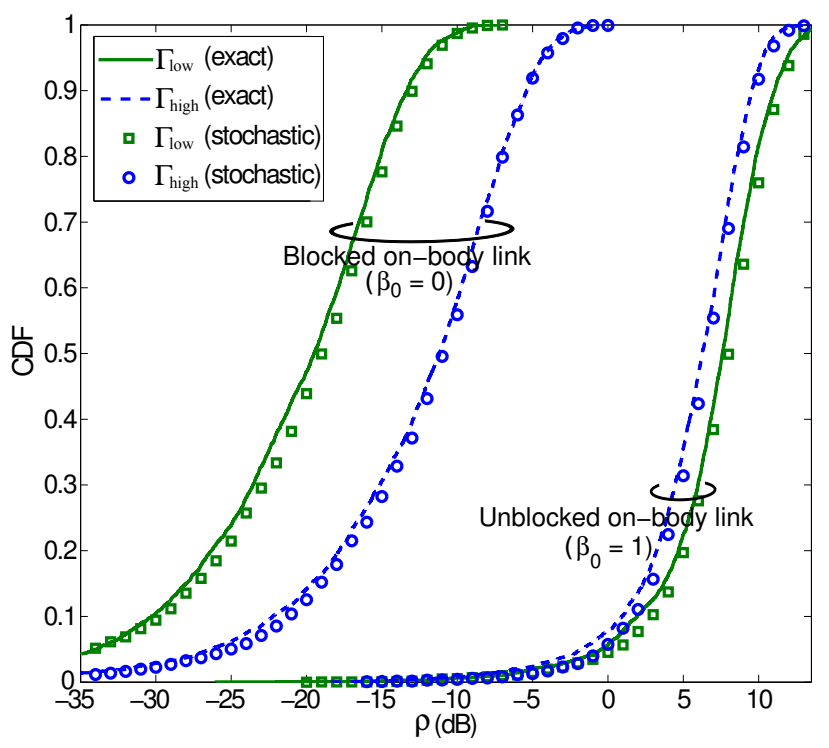

Fig. 4. CDF of SINR when the reference receiver is located at the center of $\mathcal{X}$, with $K=30$.

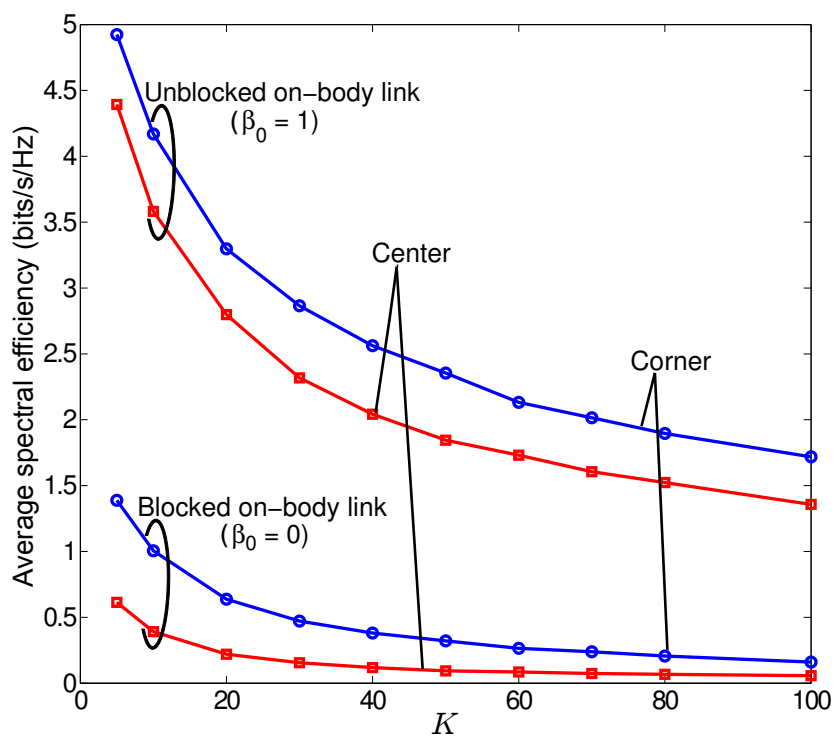

Fig. 5. Spatially averaged spectral efficiency (bits/s/Hz) with high-reflectivity surfaces.

which can be spatially averaged over the distribution of $\rho$ (dictated by all possible locations of the transmitters and the users) to obtain the average performance for a given reference receiver location.

Example 2. Shown in Fig. 5 is the spatially averaged spectral efficiency, as function of $K$, with high reflectivity surfaces $\left(\Gamma_{\text {high }}\right)$. The contrast is between center and corner receiver locations, and between unblocked and blocked on-body link.

\section{SUMMARY}

The performance of mmWave wearable networks in enclosed spaces is influenced decidedly by blockages and reflec- tions. We have proposed a simple stochastic model to account for these two effects, and validated the satisfactory behavior of this model in certain settings. Further work is needed to generalize this model (e.g., to non-rectangular spaces and higher-order signal bounces) and to extend its validation.

In terms of the impact of reflections, the examples presented lead to the following observations:

- When the direct on-body signal is unblocked, reflections are overall detrimental. The increase in interference dominates the increase in useful signal, as indicated by the degradation in performance with the increase in reflectivity (cf. Fig. 4).

- When the direct on-body signal is blocked and the intended signal is received only via reflections, increased reflectivity improves the performance yet the SINR is very low and operation might not be feasible without strong antenna gains.

As far as blockages are concerned, their probability increases with the user density, but not fast enough to fully shield receivers and hence the cumulative interference grows with the user density. Corner locations are more favorable because there is a natural protection from direct interference.

Altogether, acceptable levels of SINR are attained when direct on-body links are unblocked by the user whereasin agreement with the ray-tracing computations in [5]-the communication feasibility is called into question if on-body links are blocked and usable signal is collected only through reflections. Given this dichotomy, a more refined (non-binary) model for the on-body blockage, with partial shadowing incorporated, would be key to clarify this feasibility.

\section{REFERENCES}

[1] Juniper Research, "Smart wearable devices: Fitness, healthcare, entertainment \& enterprise 2013-2018," 2013.

[2] A. Pyattaev, K. Johnsson, S. Andreev, and Y. Koucheryavy, "Communication challenges in high-density deployments of wearable wireless devices," IEEE Wireless Commun. Mag., vol. 22, pp. 12-18, Feb. 2015.

[3] K. Venugopal, M. C. Valenti, and R. W. Heath Jr., "Interference in finitesized highly dense millimeter wave networks," in Proc. IEEE Int. Symp. Inform. Theory and its Applications, Feb. 2015.

[4] T. Rappaport et al., "Millimeter wave mobile communications for $5 \mathrm{G}$ cellular: It will work!" IEEE Access, vol. 1, pp. 335-349, May 2013.

[5] Z. Genc, U. Rizvi, E. Onur, and I. Niemegeers, "Robust $60 \mathrm{GHz}$ indoor connectivity: Is it possible with reflections?" in Proc. IEEE Veh. Technol. Conf., May 2010, pp. 1-5.

[6] Y. Niu, Y. Li, D. Jin, L. Su, and A. V. Vasilakos, "A survey of millimeter wave (mmWave) communications for 5G: Opportunities and challenges," 2015, Online: http://arxiv.org/abs/1502.07228.

[7] P. Karadimas, B. Allen, and P. Smith, "Human body shadowing characterization for 60-GHz indoor short-range wireless links," IEEE Antennas Wireless Propagat. Lett., vol. 12, pp. 1650-1653, Dec. 2013.

[8] G. George and A. Lozano, "Performance of enclosed mmWave wearable networks," 2015, Online: http://www.dtic.upf.edu/ alozano/resume/ index.html\#publications.

[9] T. Bai and R. W. Heath Jr., "Analysis of self-body blocking effects in millimeter wave cellular networks," in Proc. Annual Asilomar Conf. Signals, Syst., Comp., Nov 2014, pp. 1921-1925.

[10] T. Bai, R. Vaze, and R. W. Heath Jr., "Analysis of blockage effects on urban cellular networks," IEEE Trans. Wireless Commun., vol. 13, no. 9 , pp. 5070-5083, Sep. 2014.

[11] K. Sato et al., "Measurements of reflection characteristics and refractive indices of interior construction materials in millimeter-wave bands," in Proc. IEEE Veh. Technol. Conf., vol. 1, Jul 1995, pp. 449-453. 